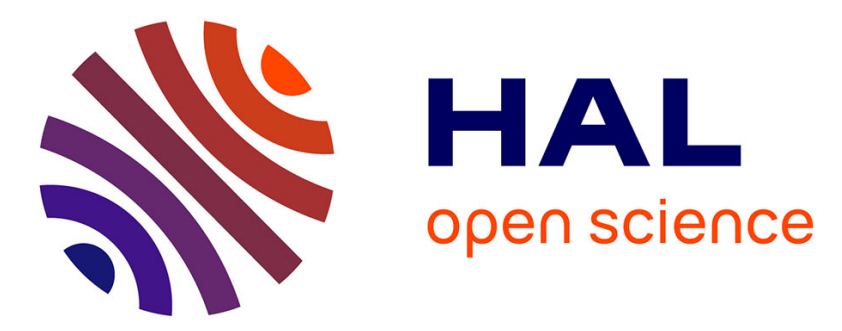

\title{
Vibroacoustic response of panels under diffuse acoustic field excitation from sensitivity functions and reciprocity principles
}

\author{
Christophe Marchetto, Laurent Maxit, Olivier Robin, Alain Berry
}

\section{- To cite this version:}

Christophe Marchetto, Laurent Maxit, Olivier Robin, Alain Berry. Vibroacoustic response of panels under diffuse acoustic field excitation from sensitivity functions and reciprocity principles. Journal of the Acoustical Society of America, 2017, 141 (6), pp.4508 - 4521. 10.1121/1.4985126 . hal-01710837

\section{HAL Id: hal-01710837 \\ https://hal.science/hal-01710837}

Submitted on 16 Feb 2018

HAL is a multi-disciplinary open access archive for the deposit and dissemination of scientific research documents, whether they are published or not. The documents may come from teaching and research institutions in France or abroad, or from public or private research centers.
L'archive ouverte pluridisciplinaire HAL, est destinée au dépôt et à la diffusion de documents scientifiques de niveau recherche, publiés ou non, émanant des établissements d'enseignement et de recherche français ou étrangers, des laboratoires publics ou privés. 


\title{
Vibroacoustic response of panels under diffuse acoustic field excitation from sensitivity functions and reciprocity principles
}

\author{
Christophe Marchetto* and Laurent Maxit \\ Univ Lyon, INSA-Lyon, Laboratoire Vibrations Acoustique, F-69621 Villeurbanne, France \\ Olivier Robin and Alain Berry \\ Groupe d'Acoustique de l'Université de Sherbrooke, \\ Université de Sherbrooke, Sherbrooke, J1K 2R1, Canada
}

(Dated: May 11, 2017)

\begin{abstract}
This paper aims at developing an experimental method to characterize the vibroacoustic response of a panel to a diffuse acoustic field excitation with a different laboratory setup than those used in standards (i.e., coupled rooms). The proposed methodology is based on a theoretical model of the diffuse acoustic field and on the measurement of the panel's sensitivity functions which characterize its vibroacoustic response to wall plane waves. These functions can be estimated experimentally using variations of the reciprocity principle which are described in the present paper. These principles can either be applied for characterizing the structural response by exciting the panel with a normal force at the point of interest or for characterizing the acoustic response (radiated pressure, acoustic intensity) by exciting the panel with a monopole and a dipole source. For both applications, the validity of the proposed approach is numerically and experimentally verified on a test case composed of a baffled simply supported plate. An implementation for estimating the sound transmission loss of the plate is finally proposed. The results are discussed and compared with measurements performed in a coupled anechoic-reverberant room facility following standards.
\end{abstract}

PACS numbers: PACS: 43.40.At, 43.40.Dx

\footnotetext{
* christophe.marchetto@usherbrooke.ca
} 


\section{INTRODUCTION}

The experimental vibroacoustic characterization of panels under a diffuse acoustic field (DAF) excitation is of great interest for the industry. This excitation is commonly used to determine the sound reduction index of panels as described in several standards using coupled reverberant-reverberant room [1] [2] or reverberant-anechoic room [3] [4] laboratory facilities. Theoretically, a DAF is defined as an infinite set of uncorrelated plane waves with uniformly distributed incidence angles. In standard laboratory measurements, this excitation is reproduced using a reverberant room and only partially corresponds to its theoretical definition, especially below the Schroeder frequency of the room where the sound field is dominated by well-defined acoustic cavity modes. Even above the Schroeder frequency, the pressure field is not perfectly homogeneous and the lack of grazing incidence plane waves has been pointed out in the literature. Inter-laboratory variations of vibroacoustic measurements in reverberant rooms can be attributed to these phenomena, but other parameters are involved such as room dimensions, niche effects, panel mounting conditions, aperture size and measuring protocols [5] [6] [7].

In this context, the aim of this study is to investigate an alternative and robust approach to experimentally characterize a panel's response to a DAF excitation by using only the theoretical model of this excitation to overcome the limitations of a reverberant room measurement discussed above. Indeed, the mathematical formulation of a panel's vibro-acoustic response when submitted to random excitations in the wavenumber domain allows estimating the system's response, at any point on the structure or in the acoustic medium, from wall-pressure cross spectral density (CSD) functions (characterizing the excitation) and from so-called 'sensitivity functions', which were introduced in [8] [9] for the analogous problem of panels excited by a turbulent flow. The latter are defined as the panel's response to wallpressure plane waves and characterize the panel's vibroacoustic behavior. The estimation of the panel's response submitted to a DAF excitation therefore only requires the experimental measurement of sensitivity functions in the acoustic wavenumber domain.

A method is proposed for estimating the sensitivity functions experimentally. Whereas the direct interpretation of the sensitivity functions would require exciting the panel by sets of wall plane waves, which is not easy from an experimental point of view, an alternative method based on a reciprocity principle is proposed. The reciprocity principle states that the 
sensitivity functions at any point on the structure or in the acoustic medium are equivalent to the panel's velocity response expressed in the wavenumber domain when the system is excited by a vibration or acoustic source at the same point. From an experimental point of view, it is then only necessary to excite the system with a vibration or acoustic source and to apply a wavenumber transform to the measured transfer function between the panel velocity and the source magnitude to obtain the sensitivity functions for a wide range of wavenumbers. Globally, the proposed experimental process consists in exciting the panel with the source of given magnitude at the point of interest. The spatial vibratory response of the panel is then measured with a scanning laser vibrometer. In a subsequent post-processing phase, a discrete 2-D wavenumber transform of the measured vibratory field is performed to deduce the sensitivity functions. Finally, using the wall-pressure model of a DAF and the previously estimated sensitivity functions, the response when the panel is excited by a DAF can be deduced at any point on the structure or in the acoustic medium. To evaluate the sound transmission loss, this process is repeated for a series of points belonging to a surface surrounding the panel to estimate the acoustic intensity at these points when the panel is excited by a DAF.

The remainder of the paper is organized as follows: the considered vibro-acoustic problem and the quantities characterizing a panel under a DAF are described in Sec. II. Then, the mathematical formulation of the vibro-acoustic problem is presented in Sec. III where the sensitivity functions appearing in the formulation are defined. An alternative interpretation of these functions based on the reciprocity principle is proposed in Sec. IV. This interpretation suggests a simple implementation for measuring the sensitivity functions. The proposed methodology for characterizing the panel response under a diffuse acoustic field is summarized in Sec. V. Numerical and experimental validations are provided in Sec. VI. Finally, a comparison with measurements performed following standards [4] is proposed in Sec. VII.

\section{VIBROACOUSTIC CHARACTERIZATION OF PANELS UNDER DIFFUSE ACOUSTIC FIELD}

Let us consider a baffled panel of surface $\Sigma_{p}$ with arbitrary boundary conditions separating two semi-infinite acoustic domains. Each of these acoustic domains is characterized by a 


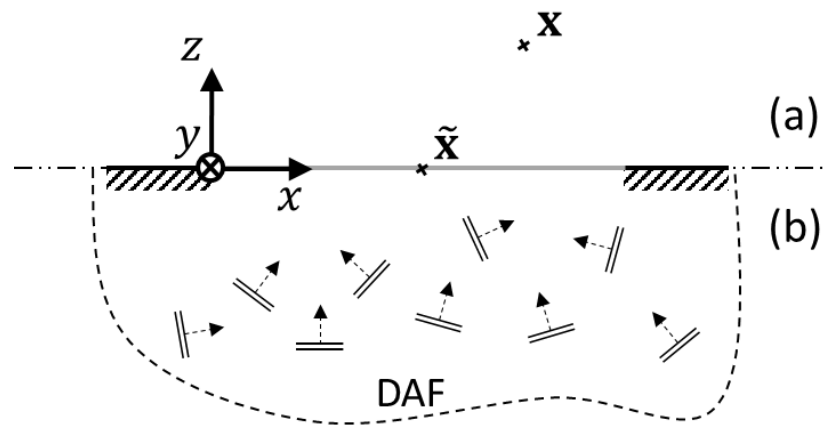

FIG. 1. Panel (gray line) and coordinate system. (a) receiving side: semi-infinite domain. (b) source side: theoretical DAF.

mass density $\rho_{0}$ and a sound velocity $c_{0}$. As shown in Fig. 1 , one supposes that a DAF is generated on one side of the panel. We define $\mathbf{x}=(x, y, z)$ the observation point in the receiving half space $z>0$ or on the panel $z=0$ and $\tilde{\mathbf{x}}=(\tilde{x}, \tilde{y})$ the excitation point on the panel surface. Both points are defined in the Cartesian coordinate system $(x, y, z)$ shown in Fig. 1.

To experimentally characterize the vibroacoustic behavior of this panel, two quantities are considered:

- the one-sided normal velocity frequency response $v$ at a given point on the panel, which for random excitations is given by the auto spectral density (ASD) function of the normal velocity $G_{v v}(\mathbf{x}, f)$,

- the transmission loss (TL) defined by

$$
T L(f)=10 \log _{10}\left(\frac{\Pi_{i n c}(f)}{\Pi_{\text {rad }}(f)}\right)
$$

where $f$ is the frequency and is considered positive.

The incident acoustic power and the radiated acoustic power are denoted $\Pi_{i n c}$ and $\Pi_{\text {rad }}$, respectively. For a DAF exciting a panel of area $\Sigma_{p}$, the incident acoustic power is theoretically given by $[10]$

$$
\Pi_{i n c}(f)=\frac{G_{p_{b} p_{b}}(f) \Sigma_{p}}{8 \rho_{0} c_{0}},
$$

where $G_{p_{b} p_{b}}(f)$ is the one-sided frequency wall-pressure ASD function and the factor 8 accounts for pressure doubling at the panel surface [11]. The radiated power of the panel 
into the receiving medium is obtained by integrating the normal active sound intensity flow passing through a virtual surface $\Sigma_{v}$ surrounding the panel

$$
\Pi_{\text {rad }}(f)=\iint_{\Sigma_{v}} \boldsymbol{I}_{a c t}(\mathbf{x}, f) \boldsymbol{n} d \mathbf{x},
$$

$\boldsymbol{n}$ being the unit exterior vector normal to $\Sigma_{v}, d \mathbf{x}$ the surface element and $\boldsymbol{I}_{a c t}(\mathbf{x}, f)$ the active sound intensity at point $\mathbf{x}$. The latter is directly related to the one-sided frequency CSD function $G_{p v_{0}}(\mathbf{x}, f)$ between the sound pressure $p$ and the particle velocity $v_{0}$ at point $\mathbf{x}$ with $[12]$

$$
\boldsymbol{I}_{a c t}(\mathbf{x}, f)=\operatorname{Re}\left\{G_{p v_{0}}(\mathbf{x}, f)\right\} .
$$

Theoretically, the radiated power is obtained by solving the formal integral in Eq. (3). For numerical and experimental applications, the integral of Eq. (3) may be approximated by discretizing the surface $\Sigma_{v}$ and using the rectangular integration rule:

$$
\Pi_{r a d}(f) \approx \sum_{\mathbf{x} \in \sigma_{v}} \boldsymbol{I}_{a c t}(\mathbf{x}, f) \boldsymbol{n} \delta \mathbf{x}
$$

where $\sigma_{v}$ represents the set of points defined on $\Sigma_{v}$ and $\delta \mathbf{x}$ is the elementary point area.

To characterize the vibration response of a panel to a DAF, it is then necessary to evaluate $G_{v v}$ at the considered point on the panel while the evaluation of $G_{p v_{0}}$ for the set of points $\sigma_{v}$ is required to estimate the TL. An approach for evaluating these quantities based on deterministic transfer functions and using a reciprocity principle is presented in the following sections.

\section{MATHEMATICAL FORMULATION OF THE VIBROACOUSTIC RESPONSE OF PANELS UNDER DIFFUSE ACOUSTIC FIELD}

Let us consider the blocked wall-pressure field $p_{b}(\tilde{\mathbf{x}}, t)$ exerted on the panel by a DAF excitation at point $\tilde{\mathbf{x}}$ as a function of time. The response of the panel at point $\mathbf{x}$ when the panel is excited by $p_{b}(\tilde{\mathbf{x}}, t)$ is denoted $\alpha(\mathbf{x}, t)$. If $\mathbf{x}$ is on the panel, $\alpha$ stands for $v$ whereas it stands for $p$ or $v_{0}$ if $\mathbf{x}$ is in the acoustic domain. This response can be expressed by the 
convolution product [13]

$$
\alpha(\mathbf{x}, t)=\iint_{\Sigma_{p}} \int_{-\infty}^{\infty} h_{\alpha / F_{n}}(\mathbf{x}, \tilde{\mathbf{x}}, t-\tau) p_{b}(\tilde{\mathbf{x}}, \tau) d \tau d \tilde{\mathbf{x}}
$$

where $h_{\alpha / F_{n}}(\mathbf{x}, \tilde{\mathbf{x}}, t)$ is the impulse response (structural velocity, acoustic pressure or particle velocity-wise) at point $\mathbf{x}$ for a normal unit force applied at point $\tilde{\mathbf{x}}$. Assuming that the random process is ergodic, the cross-correlation function $R_{\alpha \alpha^{\prime}}(\mathbf{x}, t)$ is defined by

$$
R_{\alpha \alpha^{\prime}}(\mathbf{x}, t)=\int_{-\infty}^{\infty} \alpha(\mathbf{x}, \tau) \alpha^{\prime}(\mathbf{x}, t+\tau) d \tau
$$

where $\alpha^{\prime}$ also designates $v, p$ or $v_{0}$. Introducing Eq. (6) in Eq. (7) and performing a time Fourier transform of the resulting expression of the cross-correlation function gives the spacefrequency spectrum $S_{\alpha \alpha^{\prime}}(\mathbf{x}, \omega)$, which after some manipulations (see [13] for details) can be written as

$$
S_{\alpha \alpha^{\prime}}(\mathbf{x}, \omega)=\iint_{\Sigma_{p}} \iint_{\Sigma_{p}} H_{\alpha / F_{n}}(\mathbf{x}, \tilde{\mathbf{x}}, \omega) H_{\alpha^{\prime} / F_{n}}^{*}(\mathbf{x}, \tilde{\tilde{\mathbf{x}}}, \omega) S_{p_{b} p_{b}}(\tilde{\mathbf{x}}, \tilde{\tilde{\mathbf{x}}}, \omega) d \tilde{\mathbf{x}} d \tilde{\tilde{\mathbf{x}}}
$$

where $H_{\alpha / F_{n}}(\mathbf{x}, \tilde{\mathbf{x}}, \omega)$ is the time Fourier transform of $h_{\alpha / F_{n}}(\mathbf{x}, \tilde{\mathbf{x}}, t)$ and corresponds to the panel frequency response function (velocity, pressure or particle velocity-wise) at point $\mathbf{x}$ when it is excited by a normal force $F_{n}$ applied at point $\tilde{\mathbf{x}} ; S_{p_{b} p_{b}}(\tilde{\mathbf{x}}, \tilde{\tilde{\mathbf{x}}}, \omega)$ is the time Fourier transform of the cross-correlation function of the blocked wall-pressure; finally ${ }^{*}$ is the complex conjugate. Defining the wavenumber-frequency spectrum of the wall-pressure $S_{p_{b} p_{b}}(\boldsymbol{k}, \omega)$ as the wavenumber transform of the space-frequency spectrum $S_{p_{b} p_{b}}(\tilde{\mathbf{x}}, \tilde{\tilde{\mathbf{x}}}, \omega)$, one has

$$
S_{p_{b} p_{b}}(\tilde{\mathbf{x}}, \tilde{\tilde{\mathbf{x}}}, \omega)=\frac{1}{4 \pi^{2}} \iint_{-\infty}^{\infty} S_{p_{b} p_{b}}(\boldsymbol{k}, \omega) e^{j \boldsymbol{k}(\tilde{\tilde{\mathbf{x}}}-\tilde{\mathbf{x}})} d \boldsymbol{k}
$$

where $\boldsymbol{k}=\left(k_{x}, k_{y}\right)$ is the wavevector defined in the plane $(x, y)$ and $d \boldsymbol{k}$ is the two-dimensional wavenumber element. By introducing Eq. (9) in Eq. (8) and rearranging the terms, one obtains

$$
S_{\alpha \alpha^{\prime}}(\mathbf{x}, \omega)=\frac{1}{4 \pi^{2}} \iint_{-\infty}^{\infty} H_{\alpha}(\mathbf{x}, \boldsymbol{k}, \omega) H_{\alpha^{\prime}}^{*}(\mathbf{x}, \boldsymbol{k}, \omega) S_{p_{b} p_{b}}(\boldsymbol{k}, \omega) d \boldsymbol{k}
$$

where

$$
H_{\alpha}(\mathbf{x}, \boldsymbol{k}, \omega)=\iint_{\Sigma_{p}} H_{\alpha / F_{n}}(\mathbf{x}, \tilde{\mathbf{x}}, \omega) e^{-j \boldsymbol{k} \tilde{\mathbf{x}}} d \tilde{\mathbf{x}}
$$


The $H_{\alpha}(\mathbf{x}, \boldsymbol{k}, \omega)$ functions are called the sensitivity functions [14] and characterize the vibroacoustic behavior of the panel. The function $H_{\alpha / F_{n}}(\mathbf{x}, \tilde{\mathbf{x}}, \omega)$ is the time Fourier transform $h_{\alpha / F_{n}}(\mathbf{x}, \tilde{\mathbf{x}}, t)$ and, therefore, corresponds to the transfer function between the panel velocity frequency response and the frequency spectrum of the applied effort.

The wall-pressure CSD function in the space-frequency domain of a DAF can be expressed by $[15]$

$$
S_{p_{b} p_{b}}(r, \omega)=S_{p_{b} p_{b}}(\omega) \frac{\sin \left(k_{0} r\right)}{k_{0} r},
$$

where $r=|\tilde{\mathbf{x}}-\tilde{\tilde{\mathbf{x}}}|, k_{0}=\omega / c_{0}$ is the acoustic wavenumber and $S_{p_{b} p_{b}}(\omega)$ is the wall-pressure ASD function. The space-wavenumber transform of Eq. (12) gives the wall-pressure CSD function in the wavenumber-frequency space

$$
S_{p_{b} p_{b}}(\boldsymbol{k}, \omega)=S_{p_{b} p_{b}}(\omega) \Phi_{p_{b} p_{b}}(\boldsymbol{k}, \omega),
$$

where

$$
\Phi_{p_{b} p_{b}}(\boldsymbol{k}, \omega)=\left\{\begin{array}{cc}
\frac{2 \pi}{k_{0}} \frac{1}{\sqrt{k_{0}^{2}-|\boldsymbol{k}|^{2}}} & \text { if }|\boldsymbol{k}|<k_{0} \\
0 & \text { if }|\boldsymbol{k}| \geq k_{0}
\end{array} .\right.
$$

As the wall-pressure CSD function of a DAF is null for wavenumbers larger than the acoustic wavenumber, the integration domain involved in Eq. (10) can be restricted to the wavenumbers contained in the acoustic domain (i.e., $|\boldsymbol{k}|<k_{0}$ ). Moreover, in practice, this integral is approximated considering a set of wavevectors in the acoustic domain $\Omega_{k}$ and using the rectangular integration rule. It should be stressed here that $S_{\alpha \alpha^{\prime}}(\mathbf{x}, \omega)$ is a two-sided spectrum as a function of the angular frequency. It can be related to the one-sided spectrum as a function of the frequency $G_{\alpha \alpha^{\prime}}(\mathbf{x}, f)$ by

$$
G_{\alpha \alpha^{\prime}}(\mathbf{x}, f)=4 \pi S_{\alpha \alpha^{\prime}}(\mathbf{x}, \omega) .
$$

For the sake of coherence with experiments, one-sided frequency spectra will be considered in the remainder of the article.

According to Eq. (10) and (15), the one-sided frequency ASD function of the velocity $v$ 
at point $\mathbf{x}(z=0)$ of a panel excited by a DAF can be estimated with

$$
G_{v v}(\mathbf{x}, f) \approx \frac{1}{4 \pi^{2}} \sum_{\boldsymbol{k} \in \Omega_{\boldsymbol{k}}}\left|H_{v}(\mathbf{x}, \boldsymbol{k}, \omega)\right|^{2} G_{p_{b} p_{b}}(f) \Phi_{p_{b} p_{b}}(\boldsymbol{k}, \omega) \delta^{2} \boldsymbol{k}
$$

whereas the one-sided frequency CSD function between the pressure $p$ and the particle velocity $v_{0}$ at a given point $\mathbf{x}$ into the acoustic domain can be estimated with

$$
G_{p v_{0}}(\mathbf{x}, f) \approx \frac{1}{4 \pi^{2}} \sum_{\boldsymbol{k} \in \Omega_{\boldsymbol{k}}} H_{p}(\mathbf{x}, \boldsymbol{k}, \omega) H_{v_{0}}^{*}(\mathbf{x}, \boldsymbol{k}, \omega) G_{p_{b} p_{b}}(f) \Phi_{p_{b} p_{b}}(\boldsymbol{k}, \omega) \delta^{2} \boldsymbol{k},
$$

where $\delta \boldsymbol{k}$ represents the wavenumber resolution and $G_{p_{b} p_{b}}(f)=4 \pi S_{p_{b} p_{b}}(\omega)$ is the one-sided frequency ASD function of the blocked wall-pressure.

To evaluate these two quantities, the sensitivity functions $H_{v}, H_{p}$ and $H_{v_{0}}$ for wavenumbers belonging to $\Omega_{\boldsymbol{k}}$ are thus to be determined. A direct interpretation of these sensitivity functions can be deduced from Eq. (11). Since $H_{\alpha / F_{n}}(\mathbf{x}, \tilde{\mathbf{x}}, \omega)$ is the response $\alpha$ at point $\mathbf{x}$ for a unit normal force at point $\tilde{\mathbf{x}}, H_{\alpha}$ represents the frequency response $\alpha$ at point $\mathbf{x}$ due to a wall-pressure plane wave of wavevector $-\boldsymbol{k}$ (i.e., due to the pressure field $\left.e^{-j \boldsymbol{k} \tilde{\mathbf{x}}}\right)$. This direct interpretation is depicted in Figs. 2(a), 2(c) and 2(e) for $H_{v}, H_{p}$ and $H_{v_{0}}$, respectively.

The sensitivity functions must, therefore, be estimated only at the point of interest $\mathbf{x}$ and for the set of wavevectors $\Omega_{\boldsymbol{k}}$. A large number of waves should be considered to entirely cover the acoustic wavenumber domain. Moreover, from an experimental point of view, wall-pressure plane waves cannot be easily reproduced. To circumvent these issues, another interpretation of these sensitivity functions based on the reciprocity principle is given in the next section.

\section{ALTERNATIVE INTERPRETATION OF THE SENSITIVITY FUNCTIONS}

In order to propose another interpretation of the sensitivity functions, let us consider the standard reciprocity principle which states that the response of a system is invariant with respect to the exchange of points of excitation and observed response [16]. Following the previous notation, it can be translated into

$$
H_{\alpha / F_{n}}(\mathbf{x}, \tilde{\mathbf{x}}, \omega)=H_{v / \bar{\alpha}}(\tilde{\mathbf{x}}, \mathbf{x}, \omega),
$$


where $H_{v / \bar{\alpha}}(\tilde{\mathbf{x}}, \mathbf{x}, \omega)$ is the frequency response function between the panel velocity and a source $\bar{\alpha}$, dual of $\alpha$. As the normal force $F_{n}$ is applied on the panel, the exchanged observation point is also on the panel which explains why the right-hand side of Eq. (18) remains the velocity response of the panel, regardless of $\alpha$. However the type of excitation source $\bar{\alpha}$ depends on $\alpha$ and it will be detailed below for each quantity considered for $\alpha$.

Sticking to the general case, by introducing Eq. (18) in Eq. (11) one obtains

$$
H_{\alpha}(\mathbf{x}, \boldsymbol{k}, \omega)=\iint_{\Sigma_{p}} H_{v / \bar{\alpha}}(\tilde{\mathbf{x}}, \mathbf{x}, \omega) e^{-j \boldsymbol{k} \tilde{\mathbf{x}}} d \tilde{\mathbf{x}} .
$$

The right hand side of Eq. (19) can be interpreted as the space-wavenumber transform of $H_{v / \bar{\alpha}}(\tilde{\mathbf{x}}, \mathbf{x}, \omega)$ with respect to the spatial variable $\tilde{\mathbf{x}}$. The points $\tilde{\mathbf{x}}$ become observation points on the panel surface $\Sigma_{p}$, which means that the space-wavenumber transform is performed over the vibration velocity field of the panel. To sum up, the sensitivity function $H_{\alpha}(\mathbf{x}, \boldsymbol{k}, \omega)$ may be obtained by exciting the panel with a source $\bar{\alpha}$ at point $\mathbf{x}$ and by calculating the space-wavenumber transform of the panel velocity frequency response normalized by the source frequency spectrum. This second interpretation of the sensitivity functions is now detailed for the three cases involved in the evaluation of the panel response excited by a DAF.

Case of plate velocity $(\alpha=v)$ : The reciprocity principle states [17] that the ratio of the normal velocity of the panel at point $\mathbf{x}$ over the applied normal force at point $\tilde{\mathbf{x}}$ is equal to the ratio of the normal velocity of the panel at point $\tilde{\mathbf{x}}$ over the normal force applied at point x. Eq. (18) becomes

$$
H_{v / F_{n}}(\mathbf{x}, \tilde{\mathbf{x}}, \omega)=H_{v / F_{n}}(\tilde{\mathbf{x}}, \mathbf{x}, \omega) .
$$

In this case, $\bar{\alpha}$ is a normal force and thus, the sensitivity function $H_{v}(\mathbf{x}, \boldsymbol{k}, \omega)$ is obtained by exciting the panel with a normal force at point $\mathbf{x}$ and by performing a space-wavenumber transform of the transfer function between the panel vibration velocity response and the force frequency spectrum, as illustrated in Fig. 2(b).

Case of radiated pressure $(\alpha=p)$ : Lyamshev reciprocity relations for elastic structures excited by point forces [16] indicate that the ratio of the pressure at point $\mathbf{x}$ over the applied normal force at point $\tilde{\mathbf{x}}$ is equal to the ratio of the normal velocity of the panel at 


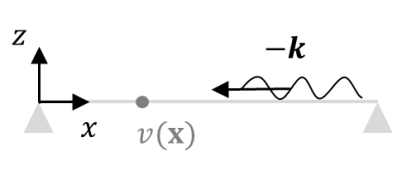

$H_{v}(\mathbf{x}, \boldsymbol{k})=v(\mathbf{x})$

(a)

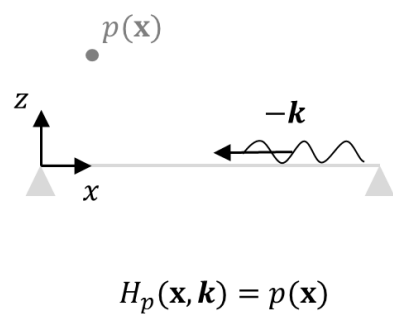

(c)

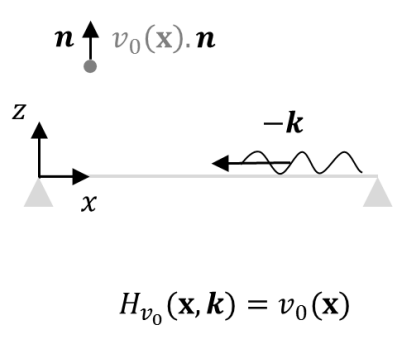

(e)

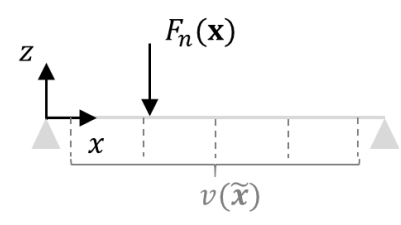

$H_{v}(\mathbf{x}, \boldsymbol{k})=\operatorname{DFT}[v(\tilde{\mathbf{x}})]$

(b)

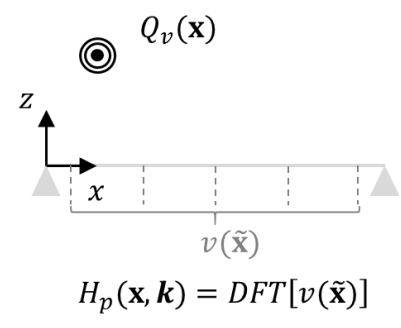

(d)

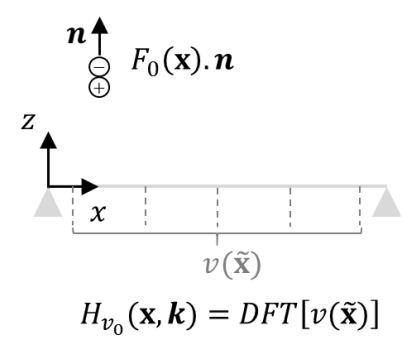

(f)

FIG. 2. Direct interpretation of the sensitivity functions: (a) $H_{v}$, (c) $H_{p}$, (e) $H_{v_{0}}$ and corresponding reciprocal interpretation (b) $H_{v}$, (d) $H_{p}$, (f) $H_{v_{0}}$. (d) and (f) see appendix for demonstration.

point $\tilde{\mathbf{x}}$ over the volume velocity $Q_{v}$ of a monopole source placed at point $\mathbf{x}$. The demonstration of this particular reciprocity relation according to Lyamshev [18] is provided in Appendix A 1 (this classical demonstration is useful to introduce reciprocity in terms of particle velocity and dipole source strength, which is demonstrated in appendix A 2). In this case $\bar{\alpha}$ is a monopole source of volume velocity $Q_{v}$ and Eq. (18) becomes

$$
H_{p / F_{n}}(\mathbf{x}, \tilde{\mathbf{x}}, \omega)=H_{v / Q_{v}}(\tilde{\mathbf{x}}, \mathbf{x}, \omega) .
$$

The sensitivity function $H_{p}(\mathbf{x}, \boldsymbol{k}, \omega)$ is therefore obtained by exciting the panel with a monopole source at point $\mathbf{x}$ and by performing a space-wavenumber transform of the panel vibration velocity response normalized by the frequency spectrum of the monopole's volume velocity, as illustrated in Fig. 2(d). 
Case of particle velocity $\left(\alpha=v_{0}\right)$ : Fahy stated that the reciprocity relationship could be extended to acoustic dipoles and particle velocities [16], however no demonstration could be found in the literature. As a point dipole can be represented by a point force injected in the fluid, the ratio of the particle velocity at point $\mathbf{x}$ over the applied normal force at point $\tilde{\mathbf{x}}$ is equal to the ratio of the normal velocity of the panel at point $\tilde{\mathbf{x}}$ over the force injected in the fluid $F_{0}$ at point $\mathbf{x}$. This latter reciprocity relation is demonstrated in Appendix A 2 . The dual source $\bar{\alpha}$ being a dipole source of force $F_{0}$ injected in the fluid, Eq. (18) now becomes

$$
H_{v_{0} / F_{n}}(\mathbf{x}, \tilde{\mathbf{x}}, \omega)=H_{v / F_{0}}(\tilde{\mathbf{x}}, \mathbf{x}, \omega)
$$

As demonstrated in Appendix A 2, the force $F_{0}$ is injected in the same direction $\boldsymbol{n}$ as the desired direction of the particle velocity $v_{0}$. One can obtain the sensitivity function $H_{v_{0}}(\mathbf{x}, \boldsymbol{k}, \omega)$ by exciting the panel with a dipole source at point $\mathbf{x}$ and by performing a space-wavenumber transform of the panel vibration velocity response normalized by the frequency spectrum of the force injected by the dipole source in the fluid, as illustrated in Fig. 2(f).

To sum up, the sensitivity functions can be obtained by exciting the system at the point of interest $\mathbf{x}$ and by performing a space-wavenumber transform of the panel velocity field. In practice, the vibratory field has to be measured on a regular grid of points denoted $\Gamma_{\tilde{\mathbf{x}}}$, using a scanning laser vibrometer, for example. The space-wavenumber transform is therefore approximated by a discrete Fourier transform. In order to avoid aliasing effects, the spatial resolution $\delta \tilde{\mathbf{x}}$ over $\Gamma_{\tilde{\mathbf{x}}}$ should be determined so that the spatial variations of the vibratory field can be correctly represented by the grid of points. For a homogeneous isotropic thin panel, $\delta \tilde{\mathbf{x}}$ should be less than or equal to a quarter of the natural flexural wavelength of the panel $\lambda_{f}$ at the highest frequency of interest. For a more complex panel, a preliminary study should be carried out to define this parameter (for instance, by using a numerical model of the panel or by using a trial and error procedure).

\section{DESCRIPTION OF THE PROPOSED METHODOLOGY}

A methodology for experimentally estimating the vibroacoutic response of a panel excited by a DAF is now presented. This methodology is based on Eqs. (16) and (17), and the second interpretation of the sensitivity functions, as described in the previous section. 
Vibration response of the panel: The methodology for evaluating the velocity ASD function $G_{v v}$ at a given point $\mathbf{x}$ of the panel $(z=0)$ can be summarized as follows:

- Excite the panel with a normal mechanical force at point $\mathbf{x}$ (for instance by using a shaker) and measure the normal velocity response of the panel at points $\tilde{\mathbf{x}} \in \Gamma_{\tilde{\mathbf{x}}}$ to determine $H_{v / F_{n}}(\tilde{\mathbf{x}}, \mathbf{x}, \omega)$,

- Perform a discrete Fourier transform of the panel velocity response $H_{v / F_{n}}(\tilde{\mathbf{x}}, \mathbf{x}, \omega)$ (with respect to $\tilde{\mathbf{x}}$ ) to obtain the sensitivity functions $H_{v}(\mathbf{x}, \boldsymbol{k}, \omega)$ at point $\mathbf{x}$ for $\boldsymbol{k} \in \Omega_{\boldsymbol{k}}$,

- Use Eqs. (16) and (14) to estimate the velocity ASD function $G_{v v}$ at point $\mathbf{x}$ under an ideal DAF excitation.

Acoustic response of the panel: The acoustic response of the panel is characterized by the TL as described in Sec. II. It can be obtained by following the next five steps:

- Excite the panel with a monopole source at a given point of interest $\mathbf{x}$ and measure the normal velocity response of the panel at points $\tilde{\mathbf{x}} \in \Gamma_{\tilde{\mathbf{x}}}$ to determine $H_{v / Q_{v}}(\tilde{\mathbf{x}}, \mathbf{x}, \omega)$,

- Excite the panel with a dipole source at a given point of interest $\mathbf{x}$ and measure the normal velocity response of the panel at points $\tilde{\mathbf{x}} \in \Gamma_{\tilde{\mathbf{x}}}$ to determine $H_{v / F_{0}}(\tilde{\mathbf{x}}, \mathbf{x}, \omega)$,

- Perform a discrete Fourier transform of the panel velocity responses obtained for both monopole and dipole cases to estimate the sensitivity functions $H_{p}(\mathbf{x}, \boldsymbol{k}, \omega)$ and $H_{v_{0}}(\mathbf{x}, \boldsymbol{k}, \omega)$ at point $\mathbf{x}$,

- Calculate the pressure - particle velocity CSD function at point $\mathbf{x}$ using Eqs. (17) and (14),

- Calculate the active sound intensity at point $\mathbf{x}$ using Eq. (4).

The five previous steps are repeated for points $\mathbf{x} \in \sigma_{v}$ (discretizing the whole virtual surface $\Sigma_{v}$ surrounding the panel) to calculate the radiated power using Eq. (5). The TL is finally deduced using Eq. (1) while the incident acoustic power is evaluated with Eq. (2). 


\section{NUMERICAL AND EXPERIMENTAL VALIDATION OF THE PROPOSED APPROACH}

\section{A. Test case description}

For numerical and experimental validation purposes a test case is considered, which consists in a rectangular thin aluminum plate, simply supported on its four edges, baffled, and submitted to a DAF excitation on one side. This baffled plate separates two semi-infinite domains filled with air $\left(\rho_{0}=1.3 \mathrm{~kg} \cdot \mathrm{m}^{-3}\right.$ and $\left.c_{0}=343 \mathrm{~m} . \mathrm{s}^{-1}\right)$. The plate's geometrical and mechanical properties are detailed in Table I. The structural loss factor $\eta_{m n}$ of the $(m, n)$ mode has been experimentally estimated using the $-3 \mathrm{~dB}$ bandwidth method on the first few resonances of the plate and is taken into account in the numerical simulations. A mean value of $\eta=0.005$ has been measured. Simply-supported boundary conditions have been chosen because they lead to a simple analytical solution of the plate equation of motion. In addition, the experimental setup proposed by Robin et al. [19] for reproducing these boundary conditions has already been validated.

The frequency range of interest is $[170,2000 \mathrm{~Hz}]$ with a frequency resolution of $0.625 \mathrm{~Hz}$. The low frequency limit is set according to the frequency response of the monopole source and the high frequency limit has been chosen arbitrarily. This frequency range is below the critical frequency of the panel, $f_{c}$, given by

$$
f_{c}=\frac{c_{0}^{2}}{2 \pi} \sqrt{\frac{\rho h}{D}}
$$

where $D=\frac{E h^{3}}{12\left(1-\nu^{2}\right)}$ is the flexural stiffness. For the considered case, $f_{c}=3867 \mathrm{~Hz}$.

In this section, we will focus on:

- the velocity sensitivity functions $H_{v}$ at point $\mathbf{x}_{M}$ of coordinates $(x=0.06 \mathrm{~m}, y=0.3 \mathrm{~m}, z=0 \mathrm{~m})$ on the plate,

- the pressure and particle velocity sensitivity functions $H_{p}$ and $H_{v_{0}}$ at point $\mathbf{x}_{N}$ of coordinates $(x=0.06 \mathrm{~m}, y=0.3 \mathrm{~m}, z=0.1 \mathrm{~m})$ into the acoustic medium. The particle velocity $v_{0}$ will be determined in direction $z$,

- the frequency response of the velocity response at point $\mathbf{x}_{M}$ and of the active intensity at point $\mathbf{x}_{N}$ in the direction $z$. 
To apply the methodology described in Sec. V, the panel velocity field has to be measured or calculated on a grid of points $\Gamma_{\tilde{\mathbf{x}}}$. In the following, a uniform mesh of $15 \times 13$ points is considered in directions $x$ and $y$ respectively and a gap of $30 \mathrm{~mm}$ along the edges is left for practical reasons. This leads to a spatial resolution of $\delta_{x}=\delta_{y}=30 \mathrm{~mm}$ and ensures at least 4 points per flexural wavelength for all frequencies of interest. The highest wavenumbers $k_{x}^{\max }$ and $k_{y}^{\max }$ that can be resolved in directions $x$ and $y$, respectively, are given by

$$
k_{x}^{\max }=k_{y}^{\max }=\frac{\pi}{\delta_{x}}=\frac{\pi}{\delta_{y}} \simeq 105 \mathrm{~m}^{-1} .
$$

These wavenumbers are well above twice the acoustic wavenumber (related to the Shannon criterion) at the highest frequency of interest (i.e., $k_{0}=37 \mathrm{~m}^{-1}$ at $2000 \mathrm{~Hz}$ ). As a consequence, the considered grid of points provides correct estimation of the sensitivity functions in the acoustic wavenumber domain $\Omega_{k}$. The wavenumber resolutions $\delta k_{x}$ and $\delta k_{y}$ in directions $x$ and $y$ respectively, are given by

$$
\delta k_{x}=\frac{2 \pi}{L_{x}} \simeq 13 \mathrm{~m}^{-1} ; \delta k_{y}=\frac{2 \pi}{L_{y}} \simeq 15 \mathrm{~m}^{-1}
$$

These wavenumber resolutions are relatively large because of the small dimensions of the panel. In order to improve the wavenumber resolution, zero-padding is used to obtain a wavenumber resolution of $0.5 \mathrm{~m}^{-1}$ along $k_{x}$ and $k_{y}$.

In order to assess the accuracy of the reciprocity approach for evaluating the panel sensitivity functions, the results obtained with this approach are compared with those obtained by considering the direct interpretation of these functions (as described in Sec. III). This comparison will allow validating the uniform mesh of $15 \times 13$ points used for the discrete spatial Fourier transform of the panel velocity field. The numerical model used for this study is described in Appendix B.

\section{B. Experimental set-up}

Fig. 3 shows the experimental setup used to measure the sensitivity functions. The plate was glued on thin blades and fixed on a frame as described in [19] to reproduce simply 


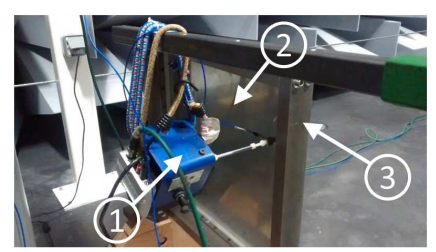

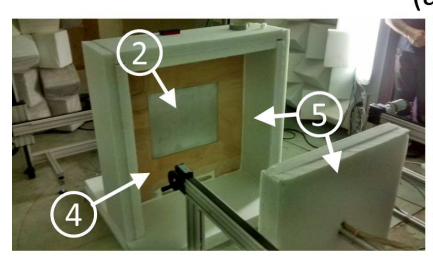

(b)

(a)

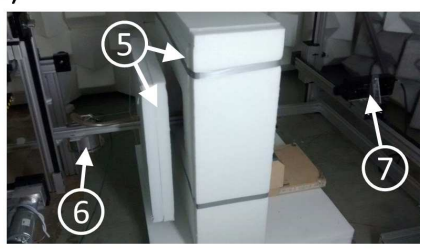

(c)

FIG. 3. Experimental setup. (a) plate excited by a shaker to determine $H_{v}$. (b) and (c) baffled plate excited by a monopole source to determine $H_{p}$ and $H_{v_{0}}$. 1 - shaker with impedance head. 2 - plate. 3 - frame. 4 - baffle. 5 - sound absorbing foam. 6 - monopole source mounted on 3-axis robot. 7 - single-point laser vibrometer mounted on 2-axis robot.

supported boundary conditions. To determine the velocity sensitivity functions $H_{v}$, the plate was excited by a normal force at point $\mathbf{x}_{M}$ of coordinates $(x=0.06 \mathrm{~m}, y=0.3 \mathrm{~m}, z=0 \mathrm{~m})$. This force was applied using a TMS SmartShaker K2007E01 with integrated amplifier, which was fed with a swept sine over the considered frequency range and the force was measured using an impedance head PCB288D01 (as shown in Fig. 3(a)). An adapter was used between the impedance head and the plate reducing the area of mechanical coupling to approximately a $5 \mathrm{~mm}$ diameter circle.

For acoustic applications, the plate was baffled in a $1 \times 1 \mathrm{~m}^{2}$ plywood panel of $2 \mathrm{~cm}$ thickness. The experiment was performed in a hemi-anechoic room and $10 \mathrm{~cm}$ thick sound absorbing foam (Decibel France Polyphone $63 \mathrm{~T}$ ) was placed on the ground and around the plate (see Figs. 3(b) and 3(c)) to avoid potential reflections and possible influence of the background noise generated by the robot used to estimate the TL (see Figs. 3(c) and section VIIB). This allowed approaching fully anechoic conditions and ideal monopole and dipole excitations.

To estimate the pressure sensitivity functions at point $\mathbf{x}_{N}$ of coordinates $(x=0.06 \mathrm{~m}, y=$ $0.3 \mathrm{~m}, z=0.1 \mathrm{~m})$, the plate was excited by a Microflown Mid-High frequency monopoleHFM source at point $\mathbf{x}_{N}$ fed with a white noise signal on the considered frequency range. The monopole source consists of a high impedance loudspeaker connected to a socket by a tube with an inner diameter of $15 \mathrm{~mm}$. The frequency range over which the source is effective 
and acts like a monopole is $[100,7000 \mathrm{~Hz}]$. The calibration of the source (volume velocity $Q_{v}$ per unit input voltage $U$ ) was obtained by measuring the radiated sound pressure $p$ at a given distance $r$ in anechoic conditions for a given input voltage $U$ and using the theoretical model of a monopole in free field for the relation between $p$ and $Q_{v}$. The effect of the tube on the frequency response has thereby been accounted for.

To estimate the particle velocity sensitivity functions at point $\mathbf{x}_{N}$ in the direction $z$, the response of the plate to a dipole source has been reconstructed by exciting the plate with the same monopole source, still fed with a white signal and moved from the previous position by a distance $d=3 \mathrm{~cm}$ in the direction of the injected force (in this case $z$ ). Two monopoles close to each other and out of phase have been thereby reconstructed by subtracting the measured transfer functions $H_{v / Q_{v}}$. A preliminary experimental study in free field conditions and using the plate was performed to determine an appropriate separation $d$. It showed that below a separation of $0.5 \mathrm{~cm}$, the vibration fields induced for both positions of the monopole source were not sufficiently different to be noticeably measured. On the other hand, above a separation of $5 \mathrm{~cm}$, the directivity of the reconstructed dipole did not match that of a theoretical dipole. A value of $3 \mathrm{~cm}$ for $d$ appeared to be an optimal value for the present case. It should be noted that the condition $k_{0} d<<1$ does not hold at the highest frequencies. However, the induced vibrations were in accordance with the response of a plate to a theoretical dipole. Since the numerical and experimental sensitivity functions and pressure - particle velocity CSD functions are in good agreement (see next section), these results validate the experimental method for reconstructing a dipole source.

According to the methodology described in Sec. V, the vibratory response of the panel has been measured on the grid of $15 \times 13$ points with a single point Polytec laser vibrometer for each case of the excitation (i.e., force, monopole, dipole). Also, in each case, the time Fourier transform was directly performed in the post-processing software with ten linear averages. 

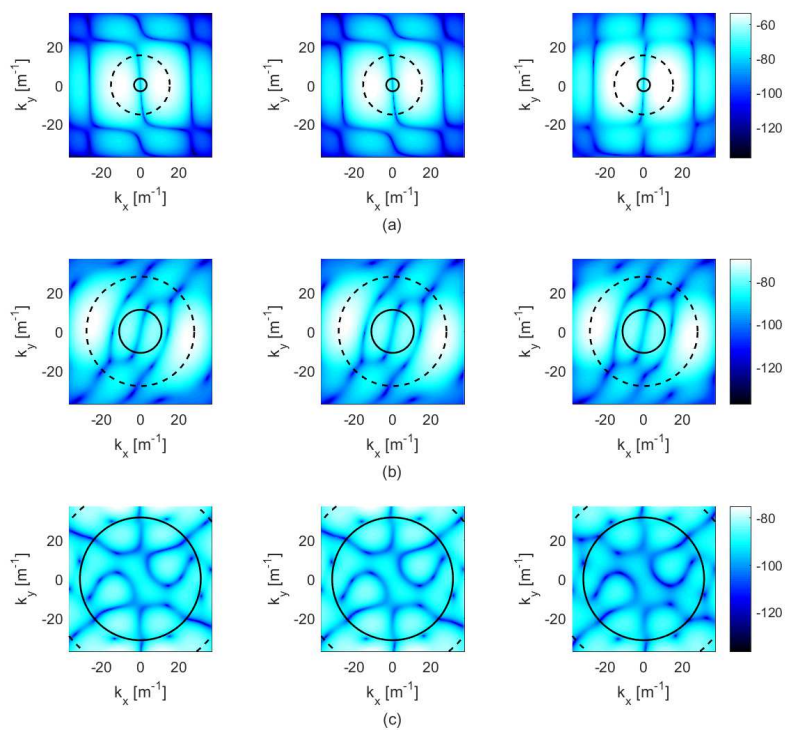

FIG. 4. Velocity sensitivity functions at point $\mathbf{x}_{M},\left|H_{v}\right|^{2}\left(\mathrm{~dB}\right.$, ref. $\left.1 \mathrm{~m}^{2} \cdot \mathrm{s}^{-2}\right)$ : direct calculation (left), numerical reciprocity approach (middle), experimental reciprocity approach (right). (a) $f=178 \mathrm{~Hz}$. (b) $f=600 \mathrm{~Hz}$. (c) $f=1710 \mathrm{~Hz}$. - - -, circle of radius $k_{f}$. - , circle of radius $k_{0}$.

\section{Comparison between numerical and experimental results}

\section{Sensitivity functions}

Fig. 4 shows the velocity sensitivity functions $H_{v}$ obtained with the direct calculation and the reciprocal approach using numerical and experimental data, respectively. They are provided for three different frequencies, the lowest corresponding to the $(2,1)$ vibration mode frequency (Fig. 4(a)) and the two others being off-resonance cases (Figs. 4(b) and 4(c)). The product of sensitivity functions $H_{p}$ and $H_{v_{0}}^{*}$, which is involved in the expression of $G_{p v_{0}}$ (Eq. (17)), is shown in Fig. 5 at the same frequencies. The circles of radius $k_{0}$ and

$k_{f}=\sqrt{\frac{2 \pi f_{c}}{c_{0}} k_{0}}$, corresponding to the acoustic and flexural natural wavenumbers respectively, are also indicated in Figs. 4 and 5.

In Fig. 4 and 5, results obtained by simulating numerically the direct and the reciprocity methods match perfectly. This validates the grid of points considered on the panel and the use of zero-padding to improve the wavenumber resolution without affecting the results. It also validates the method described above to reconstruct a dipole from two monopole sources.

In Fig. 4 and 5, the numerical and experimental results are generally in good agreement. 

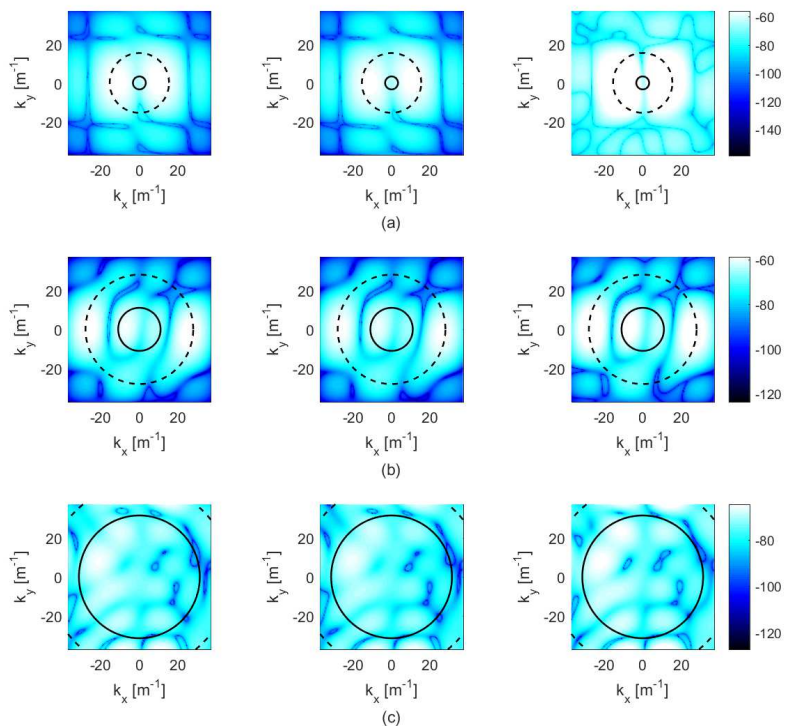

FIG. 5. Product of sensitivity functions at point $\mathbf{x}_{N},\left|\operatorname{Re}\left\{H_{p} \times H_{v_{0}}^{*}\right\}\right|\left(\mathrm{dB}\right.$, ref. 1 Pa.m.s $\left.{ }^{-1}\right)$ : direct calculation (left), numerical reciprocity approach (middle), experimental reciprocity approach (right). (a) $f=178 \mathrm{~Hz}$. (b) $f=600 \mathrm{~Hz}$. (c) $f=1710 \mathrm{~Hz}$. - - -, circle of radius $k_{f}$. - , circle of radius $k_{0}$.

One can observe that the sensitivity functions are slightly overestimated experimentally at the $(2,1)$ vibration mode frequency compared to the numerical results. This can be explained by the fact that the modal damping loss factor has been estimated from the response of the plate to a shaker excitation. The added mass from the shaker possibly had an influence on the evaluation of the damping of the $(2,1)$ mode.

A good agreement is particularly noticed within the acoustic wavenumber circle (delineated by a full line). Again, only values in the acoustic wavenumber domain contribute to the plate's vibroacoutic response to a DAF. However, the sensitivity functions are also correctly estimated experimentally for wavenumbers higher than the acoustic wavenumber.

\section{Plate velocity ASD function}

The velocity ASD function $G_{v v}(\mathbf{x}, f)$ at point $\mathbf{x}_{M}$ of the panel excited by a DAF with a unit wall-pressure ASD function $\left(G_{p_{b} p_{b}}(f)=1 \mathrm{~Pa}^{2} \cdot \mathrm{Hz}^{-1}\right)$ has been estimated using Eq. (16) and the three previously described sensitivity functions. Fig. 6(a) compares the results obtained with the direct calculation and the numerical reciprocity approach. The two curves are perfectly superimposed, showing that the sensitivity functions obtained with the two 

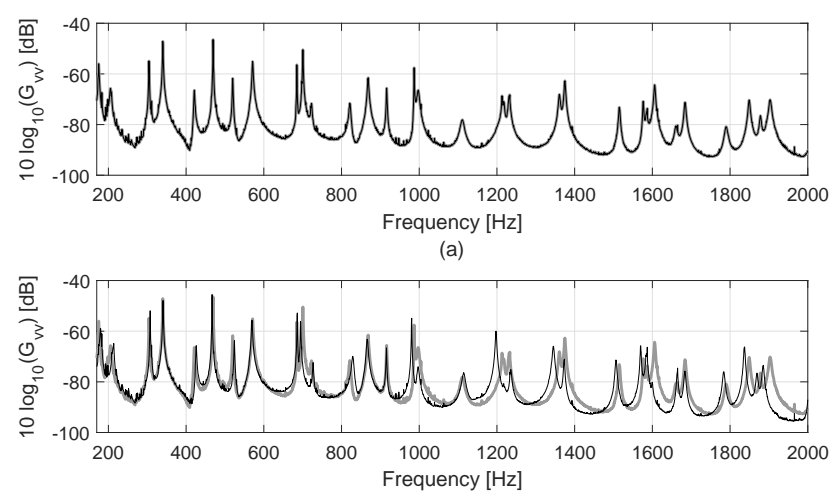

(b)

FIG. 6. Velocity ASD functions $G_{v v}\left(\mathrm{~dB}\right.$, ref. $1 \mathrm{~m}^{2} \cdot \mathrm{s}^{-2} \cdot \mathrm{Hz}^{-1}$ ). (a) direct calculation (bold gray line) vs. numerical reciprocity approach (light black line). (b) numerical reciprocity approach (bold gray line) vs. experimental reciprocity approach (light black line).

approaches are essentially identical in the acoustic domain and on the whole considered frequency range. The small noticeable peaks between resonance frequencies of the plate on both curves are attributable to the wavenumber sampling in Eq. (16) which induces errors especially as $|\boldsymbol{k}|$ approaches $k_{0}$, in which case the DAF wall-pressure CSD goes to infinity (see Eq. (14)).

Fig. 6(b) compares the results obtained numerically and experimentally while considering the reciprocity method to estimate the sensitivity functions. A good agreement is observed between the two results, which experimentally validates the proposed methodology for the considered test case. Slight shifts of the resonance peaks in the high frequency range are noticed. They can be explained by small differences between the experimental and the theoretical boundary conditions of the panel or more likely by the added mass from the shaker.

\section{Pressure - particle velocity CSD function}

Fig. 7(a) shows the real part of the pressure - particle velocity CSD function $G_{p v_{0}}(\mathbf{x}, f)$ at point $\mathbf{x}_{N}$ when the plate is excited by a $\operatorname{DAF}\left(G_{p_{b} p_{b}}(f)=1 \mathrm{~Pa}^{2} \cdot \mathrm{Hz}^{-1}\right)$. These results have been obtained using the direct calculation and the reciprocal approach for the sensitivity functions. Again, the two curves are in good agreement, which shows that the sensitivity functions $H_{p}$ and $H_{v_{0}}$ are properly determined on the entire frequency range using the 

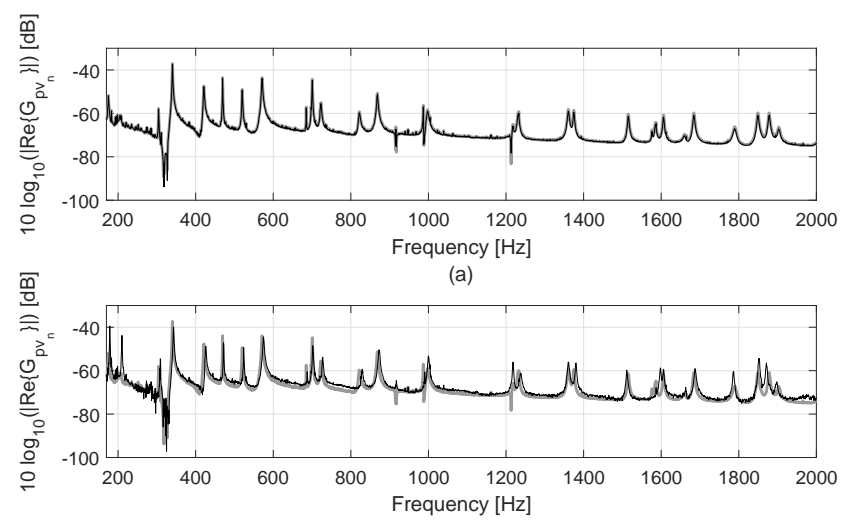

(b)

FIG. 7. Pressure - particle velocity CSD functions $\operatorname{Re}\left\{G_{p v_{0}}\right\}\left(\mathrm{dB}\right.$, ref. $\left.1 \mathrm{~W}^{2} \cdot \mathrm{m}^{-2} \cdot \mathrm{Hz}^{-1}\right)$. (a) direct calculation (bold gray line) vs. numerical reciprocity approach (light black line). (b) numerical reciprocity approach (bold gray line) vs. experimental reciprocity approach (light black line).

reciprocity principle.

The experimental and theoretical pressure - particle velocity CSD functions are compared in Fig. 7(b). Their good agreement shows that the active sound intensity is correctly estimated in this situation using the reciprocity approach. Furthermore, the experimental monopole and dipole excitations correctly reproduce the theoretical conditions. The resonance peaks are better estimated compared to those obtained to estimate the plate's velocity ASD function in Fig. 6(b), particularly at high frequencies. This can be possibly explained by the accuracy in positioning the source which, for the experiment with the shaker was done manually (subject to more errors) whereas for the acoustic applications, it was controlled with a robot allowing a higher accuracy. A more likely explanation is the dynamic influence of the mass added with the shaker, which explains the slight shifts of the resonance peaks in Fig. 6(b).

\section{COMPARISON WITH REVERBERANT ROOM MEASUREMENTS}

The proposed approach is finally compared with measurements performed at the University of Sherbrooke transmission loss facility (coupled reverberant-anechoic rooms) using a plate similar to the one used in the previous section (similar dimensions, material and boundary conditions) and following test standard ASTM E2249-02 (2016) [4]. The reverberant room has a volume of approximately $140 \mathrm{~m}^{3}\left(7.5 \times 6.2 \times 3 \mathrm{~m}^{3}\right)$, and the Schroeder frequency 
of the room, above which the sound field can be considered diffuse, is approximately $410 \mathrm{~Hz}$. The plate was mounted in an existing niche between the coupled anechoic-reverberant rooms (the panel being flush mounted on the reverberant room side). A double-wall structure with mechanical decoupling was then built around the plate to prevent acoustic leaks and flanking paths, as described in [19]. A loudspeaker fed with a white noise signal excited the reverberant chamber.

\section{A. Panel velocity response}

A first experiment in the transmission loss facility was carried out to evaluate the vibratory response of the panel under a DAF. A Polytec scanning laser vibrometer placed on the anechoic side was used to measure the plate velocity ASD function. A $9 \times 9$ microphones array $(1 / 4$ inch $B \& K$ 4957) separated by $10 \mathrm{~cm}$ in directions $x$ and $y$ was centered to the plate and used to directly measure wall-pressure fluctuations $1 \mathrm{~cm}$ away from the plate. An average sound pressure level over all 81 microphones was then calculated to evaluate a mean wall-pressure ASD function $\bar{G}_{p_{b} p_{b}}(f)$ on the reverberant side.

The sensitivity functions of this second plate were estimated experimentally using the reciprocity method. Some differences with the sensitivity functions of the plate considered in Sec. VI (not shown here) indicate that the positioning of the force applied with the shaker is not exactly at the considered point $\mathbf{x}_{M}$. Indeed, the position has a significant influence on the vibratory response, particularly at high frequencies where the mode shapes get more complex. The mounting base of the force sensors also has a finite diameter of approximately $5 \mathrm{~mm}$, which makes the applied force not perfectly punctual. However, the modal frequencies correspond to the theory for both plates. The velocity ASD function was calculated using Eq. (16) and (14) whilst including the measured wall-pressure ASD function $\bar{G}_{p_{b} p_{b}}(f)$.

The plate velocity ASD function measured in the reverberant room at point $\mathbf{x}_{M}$ is compared to the result obtained with the proposed method in Fig. 8. The two obtained responses are in good agreement up to $800 \mathrm{~Hz}$. Above this frequency, differences are noticeable and can be explained with:

- the inaccuracy in the positioning of point $\mathbf{x}_{M}$ where the velocity ASD function is measured in the reverberant chamber (corresponding to the point force position in the reciprocity method) whose influence increases with the frequency, 


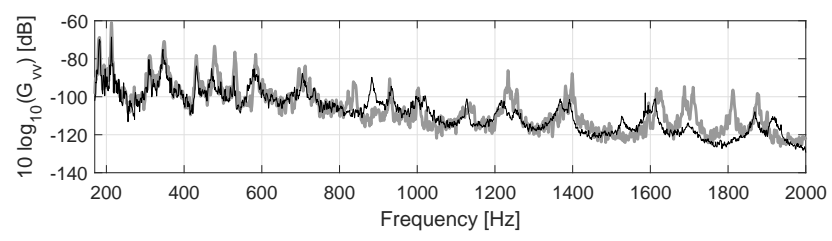

FIG. 8. Velocity ASD functions $G_{v v}\left(\mathrm{~dB}\right.$, ref. $\left.1 \mathrm{~m}^{2} \cdot \mathrm{s}^{-2} \cdot \mathrm{Hz}^{-1}\right)$ : reverberant room measurements (bold gray line) vs. experimental reciprocity approach (light black line).

- the deviation of the pressure field in reverberant room to an ideal DAF. An analysis of the pressure field measured with the microphone array shows spatial variations in contradiction with the assumptions of a perfect DAF. Moreover, it is well known that a reverberant chamber has difficulty creating grazing incidence waves. The absence of grazing incidence waves can hardly be quantified as it varies from one reverberant room to another. Some authors suggest corrections on the theoretical model of the excitation to better represent the actual excitation in a reverberant room [20] [21].

\section{B. Sound transmission loss}

A second experiment in the transmission loss facility was conducted for estimating the plate TL. A 1/2 inch Bruel \& Kjaer rotating microphone was used to measure the spatiallyaveraged sound pressure level $L_{p}$ in far radiation field. The average sound intensity level $L_{I}$ was measured on the anechoic side by using a Bruel \& Kjaer sound intensity probe composed of two $1 / 2$ inch microphones with a $12 \mathrm{~mm}$ spacing. The sound intensity probe was manually moved $5 \mathrm{~cm}$ away from the plate to scan over a parallel surface identical to the plate area, as described in [4] for the case of a plate flush mounted on the source side. The transmission loss of the structure is given by $T L=L_{p}-L_{I}-6$ [22]. An illustration of this experiment and the considered virtual surface is given in Fig. 9(a).

In addition, the proposed methodology described in Sec. V for estimating the TL was applied. A numerical study on the definition of the virtual surface $\Sigma_{v}$ (which is not detailed in this paper) showed that considering a virtual surface equal to the plate area and positioned $5 \mathrm{~cm}$ away from it would result in a slight overestimation of the TL, particularly at high frequencies. This overestimation is due to sound intensity levels outside the virtual surface $\Sigma_{v}$ that are thus not taken into account in the calculation of the radiated power. The 


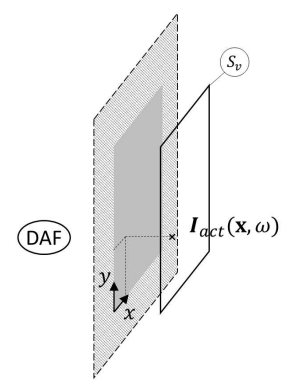

(a)

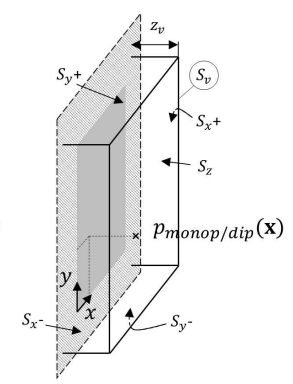

(b)

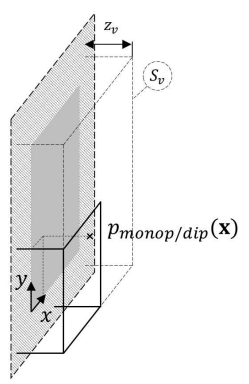

$(c)$

FIG. 9. Illustration of the virtual surface $\Sigma_{v}$ considered for estimating the radiated power. (a) in the transmission loss facility. (b) with the reciprocal approach. (c) with the reciprocal approach considering the symmetry properties of the system.

virtual surface $\Sigma_{v}$ over which the active sound intensity should be estimated to obtain the radiated power should enclose the plate entirely. Therefore, the considered surface $\Sigma_{v}$ was decomposed into 5 surfaces: (a), the surface $S_{z}$ which is directly in the front of the plate, of dimensions $0.66 \mathrm{~m} \times 0.6 \mathrm{~m}$ and positioned at $z_{v}=0.05 \mathrm{~m}$; (b), the four lateral surfaces (denoted $S_{x} \pm, S_{y} \pm$ as shown in Fig. 9(b)) to enclose the whole panel. The active sound intensity on $S_{z}$ was calculated on a grid of $12 \times 10$ points uniformly distributed along $x$ and $y$, respectively. The sound intensity was only calculated on 10 aligned points on $S_{x} \pm$ uniformly distributed along $y$ and 12 aligned points on $S_{y} \pm$ uniformly distributed along $x$. In both case, the points were positioned at $z_{v} / 2$. Note that in the reciprocal approach, the direction of the active sound intensity is defined by the direction of the force injected by the dipole (see Fig. 2(f)). To determine the active sound intensity at point $\mathbf{x}$ using the reciprocity principle, the plate was excited successively by a monopole and dipole source at point $\mathbf{x}$.

To reduce the number of excitation points and the measurement time, the symmetries of the system (with respect to $x=L_{x} / 2$ and $y=L_{y} / 2$ ) were considered. Only the points belonging to a fourth of $\Sigma_{v}$, as illustrated in Fig. 9(c), were considered, leading to a total of 40 positions of excitation as compared to 160 in Fig. 9(b). The experiment was performed in an anechoic room, using two translating robots to automate the process (see Figs. 3(b) and $3(\mathrm{c}))$. One robot was used to move the monopole source over each point $\sigma_{v}$ defining the discretized surfaces (considering the above symmetry) and the other robot was used to move a Polytec single-point laser vibrometer measuring the panel velocity on a $15 \times 13$ point 


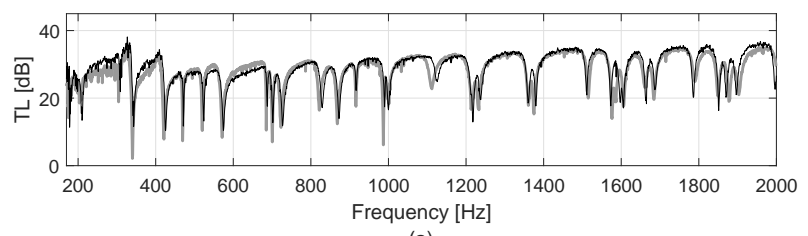

(a)

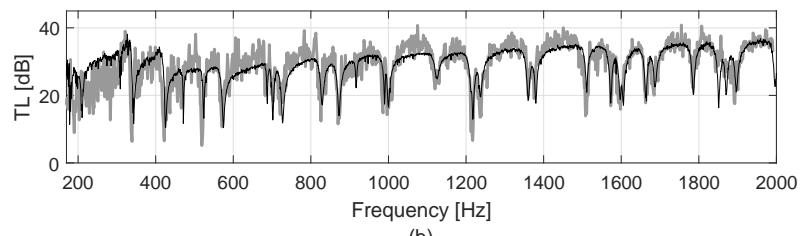

(b)

FIG. 10. Transmission loss (dB). (a) numerical approach (bold gray line) vs. experimental reciprocity approach (light black line). (b) transmission loss facility (bold gray line) vs. experimental reciprocity approach (light black line).

mesh.

As a reference to compare the following numerical and experimental results, a numerical model has been established to accurately evaluate the radiated power from wall pressure and wall velocity values. At the wall, the particle velocity sensitivity function $H_{v_{0}}$ normal to the plate is equal to the plate velocity sensitivity function $H_{v}$. Expressing it in the wavenumber domain allows using the wavenumber relation between the acoustic pressure and the particle velocity [23] and thus determining $H_{p}$ at the wall. Finally, by expressing Eqs. (4), (5) and (17) in the wavenumber domain and using $H_{p}$ and $H_{v_{0}}$ determined at the wall, one obtains the radiated power directly at the wall.

Fig. 10(a) shows experimental results obtained based on the reciprocity method, while considering the virtual surface $\Sigma_{v}$ described at the beginning of this section and illustrated in Figs. 9(b) and 9(c), versus simulation results for which the radiated power was evaluated at the panel surface. The curves match very well on the whole frequency range. This demonstrates that the proposed reciprocity approach accurately reproduces the theoretical TL for the considered test case. Moreover, it validates the definition of the virtual surface $\Sigma_{v}$ considered in the experiment.

The experimental results obtained with the reciprocity principle are compared in Fig. 10(b) to the experimental results obtained in the transmission loss facility. The transmission loss facility results are noisier than those derived from the reciprocity principle. A good agreement is however noticed in general. Relatively small differences are noticeable above the 
Schroeder frequency $(410 \mathrm{~Hz})$. These could be explained by the non-perfectly diffuse character of the sound field in the reverberant room. On the other hand, below $410 \mathrm{~Hz}$ the TL measured in the transmission loss facility is on average $6.5 \mathrm{~dB}$ lower than the one obtained with the reciprocal approach. This is explained by the modal behavior of the reverberant room below the Schroeder frequency, which enhances the non diffuse character of the incident sound field.

\section{CONCLUSION}

In this paper, a methodology for characterizing the response of flat panels to a diffuse acoustic field excitation without using a reverberant room was proposed. This approach is based on the mathematical formulation of the random excitation problem in the wavenumber domain. This formulation indicates that the panel's response at point $\mathbf{x}$ (on the panel or in the acoustic medium) to a random field depends on two quantities in the wavenumber domain: the wall-pressure cross spectral density function of the excitation and on so-called 'sensitivity functions' at point $\mathbf{x}$ which characterize the panel. Using the reciprocity principle, it has been shown that these functions can be determined from the panel velocity field in the wavenumber domain when the system is excited by a source of unit amplitude at the point of interest $\mathbf{x}$. The sensitivity functions can be estimated easily by experiment based on the reciprocal interpretation.

The proposed approach avoids the use of a reverberant room to determine the sound transmission loss factor and vibration response of plane panels under a diffuse field excitation. As the excitation is represented by an analytical model, this approach can be applied to experimentally characterize the vibroacoustic response of a panel to an ideal diffuse acoustic field. It should however be underlined that the main limitations of the proposed approach rely on the assumptions of the mathematical formulation of the problem: the system should be linear (i.e., elastic material, small deformations) and time invariant, and the condition of a baffled panel in an anechoic environment should be verified (particularly for acoustic applications). It offers however a large field of applications.

From a practical point of view, the vibration response of a panel to a diffuse field excitation can be easily estimated using a mechanical source of effort and a vibration measuring device to determine the vibratory response of the panel (in this study, a shaker and a scanning laser 
vibrometer were used). In this particular case, the use of a baffle is not crucial, because no acoustic excitation are considered.

For acoustic applications, the condition of baffled panel in an anechoic environment is fundamental to agree with the assumption of blocked pressure. Monopole and dipole sources are required to determine the radiated pressure and the particle velocity, respectively. It can be quite challenging to experimentally reproduce those sources. In this study, a monopolelike source was used and moved from a certain distance to represent two monopoles close to each other and out of phase. A dipole source could thereby be reproduced. For the determination of the sound transmission loss factor, the monopole and dipole excitations have to be applied on several points discretizing a virtual surface, which encloses the whole panel. For each position of the excitation, the vibration response of the entire panel has to be measured. In this study, two translating robots were used to automate the process. As opposed to measurements in a transmission loss facility, this experiment was highly timeconsuming. However, with the recently developed vibration measuring techniques (such as optical measurement), the time of experiment could be largely reduced.

To conclude, the method has been validated numerically and experimentally for the considered test case. Comparisons of numerical and experimental results have shown that the sensitivity functions have been well estimated both inside and outside the acoustic circle in the wavenumber domain. A good agreement between numerical and experimental results has also been obtained whether for the velocity spectrum at a point on the panel or for the sound intensity spectrum at a point in the acoustic domain. An application of the proposed methodology for estimating the sound transmission loss of the plate has been presented and the results have been compared with standard measurements in a coupled room facility. In the near future, the method will be extended to the characterization of panels excited by a turbulent boundary layer.

\section{ACKNOWLEDGMENTS}

This work was supported by the Labex CeLyA of Université de Lyon, operated by the French National Research Agency (ANR-10-LABX-0060/ANR-11-IDEX-0007).

Special thanks must go to Mr Patrick Blachier for his considerable contribution to all 
experimental setups required to perform this study.

[1] ISO 10140-2:2010 Acoustics - Laboratory measurement of sound insulation of building elements - Part 2: Measurement of airborne sound insulation (International Standard Organization, Geneva, Switzerland, 2010).

[2] ASTM E90-09 Standard Test Method for Laboratory Measurement of Airborne Sound Transmission Loss of Building Partitions and Elements (ASTM International, West Conshohocken, PA, 2009).

[3] ISO 15186-1:2000 Acoustics - Measurement of sound insulation in buildings and of building elements using sound intensity - Part 1: Laboratory measurements (International Standard Organization, Geneva, Switzerland, 2000).

[4] ASTM E2249-02 (2016) Standard Test Method for Laboratory Measurement of Airborne Sound Transmission Loss of Building Partitions and Elements Using Sound Intensity (ASTM International, West Conshohocken, PA, 2016).

[5] T. Bravo, S. J. Elliott, "Variability of low frequency sound transmission measurements", J. Acoust. Soc. Am. 115(6), 2986-2997 (2004).

[6] A. Dijckmans, C. Vermeir, "Numerical investigation of the repeatability and reproductibility of laboratory sound insulation measurements", Acta Acust. United Ac. 99, 421-432 (2013).

[7] N. Garg, L. Gandhi, A. Kumar, P. Kumar, P. K. Saini, "Measurement uncertainty in airborne sound insulation and single-number quantities using sound pressure and sound intensity approaches", Noise Control Eng. J. 64(2), 153-169 (2016).

[8] W. K. Bonness, D. E. Capone, S. A. Hambric, "Low-wavenumber turbulent boundary layer wall-pressure measurements from vibration data on a cylinder in pipe flow", J. Sound Vib. 329, 4166-4180 (2010).

[9] S. A. Hambric, Y. F. Hwang, W. K. Bonness, "Vibrations of plates with clamped and free edges excited by low-speed turbulent boundary layer flow", J. Fluid Struct. 19, 93-110 (2004).

[10] O. Robin, A. Berry, S. Moreau, "Experimental vibroacoustic testing of plane panels using synthesized random pressure fields", J. Acoust. Soc. Am. 135(6), 3434-3445 (2014).

[11] J-D. Chazot, O. Robin, J-L. Guyader, N. Atalla, "Diffuse Acoustic Field Produced in Reverberant Rooms: A Boundary Diffuse Field Index", Acta Acust. United Ac. 102, 503-516 
(2016).

[12] F. Fahy, "Sound Intensity", pp. 96, Elsevier Applied Science, London (1989).

[13] C. Maury, P. Gardonio, S. J. Elliott, "A wavenumber approach to modelling the response of a randomly excited panel, part 1: general theory", J. Sound Vib. 252(1), 83-113 (2002).

[14] Y. K. Lin, "Probabilistic theory of structural dynamics", pp. 207, McGraw-Hill, New York (1967).

[15] R. K. Cook, R. V. Waterhouse, R. D. Berendt, S. Edelman, M. C. Thompson Jr, "Measurement of correlation coefficients in reverberant sound fields", J. Acoust. Soc. Am. 27(6), 1072-1077 (1955).

[16] F. J. Fahy, "Some Applications of the Reciprocity Principle in Experimental Vibroacoustics", Acoustical Physics 49(2), 217-229 (2003).

[17] L. Maxit, V. Denis, "Prediction of flow induced sound and vibration of periodically stiffened plates”, J. Acoust. Soc. Am. 133(1), 146-160 (2013).

[18] L. M. Lyamshev, "A method for solving the proble; of sound radiation by thin elastic shells and plates", Sov. Phys. Acoust. 102(5), 122-124 (1958).

[19] O. Robin, J-D. Chazot, R. Boulandet, M. Michau, A. Berry, N. Atalla, "A plane and thin panel with representative simply supported boundary conditions for laboratory vibroacoustic test", Acta Acust. United Ac. 102(1), 170-182 (2016).

[20] H. -J. Kang, J. -G. Ih, H. -S. Kim, J. -S. Kim "An experimental investigation on the directional distribution of incident energy for the prediction of sound transmission loss", Applied Acoustics 63, 283-294 (2002).

[21] N. H. Schiller, A. R. Allen, "Assessment of analytical predictions for diffuse field sound transmission loss", In proceedings of the 44th InterNoise Congress, Paper No. IN15_257, pp. 59715982, San Francisco, California, USA (2015).

[22] R. W. Guy, A. De Mey, "Measurement of sound transmission loss by sound intensity", Canadian Acoustics 13(2), 25-44 (1985).

[23] E. G. Williams, J. D. Maynard, "Numerical evaluation of the Rayleigh integral for planar radiators using the FFT", J. Acoust. Soc. Am. 72(6), 2020-2030 (1982).

[24] A. D. Pierce, "Acoustics: An Introduction to Its Physical Principles and Applications", pp. 165-167, Acoustical Society of America, New York (1989). 


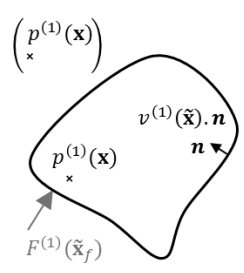

(a)

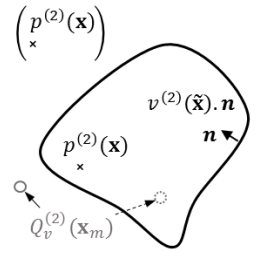

(b)

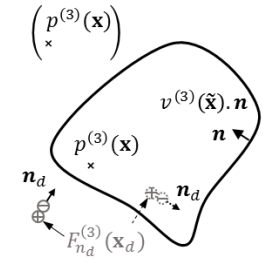

(c)

FIG. 11. Illustration of the three considered vibroacoustic problems of an elastic structure excited by: (a) a normal force at point $\tilde{\mathbf{x}}_{f}$, (b) a monopole source at point $\mathbf{x}_{m}$ (internal or external to the structure), (c) a dipole source at point $\mathbf{x}_{d}$ (internal or external to the structure).

Appendix A: Acoustic reciprocity principles: mathematical formulation of the different vibroacoustic problems

Let's consider a thin elastic structure in an acoustic medium of fluid density $\rho_{0}$ where sound waves propagate at a certain speed $c_{0}$. The vibroacoustic response of a structure at any given point $\mathbf{x}=(x, y, z)$ (belonging to the acoustic medium or the structure) is studied for three separate loadings (as illustrated in Fig. 11):

1. a normal point force at point $\tilde{\mathbf{x}}_{f}$,

2. a monopole source at point $\mathbf{x}_{m}$,

3. a dipole source at point $\mathbf{x}_{d}$,

where points belonging to the structure are denoted $\tilde{\mathbf{x}}$.

Any kind of thin elastic structure can be considered; and the excitation and observation point $\left(p^{(i)}(\mathbf{x}), i=1,2\right.$ or 3$)$ can either be internal or external to the structure. It is also assumed that there is no other acoustic loading on the other side of the structure. The monopole source can be introduced in the Helmholtz equation by a Dirac function at point $\mathbf{x}_{m}$. As a dipole source is defined by two monopoles separated by a distance $d$ (supposedly small compared to the acoustic wavelength) and out of phase, a dipole source is introduced by the gradient of a Dirac at point $\mathbf{x}_{d}$ in direction $\mathbf{n}_{d}$. Sticking to the above numbering for each load case, the Helmholtz equations are given by

$$
\Delta p^{(1)}(\mathbf{x})+k_{0}^{2} p^{(1)}(\mathbf{x})=0,
$$




$$
\begin{gathered}
\Delta p^{(2)}(\mathbf{x})+k_{0}^{2} p^{(2)}(\mathbf{x})=-j \omega \rho_{0} Q_{v}^{(2)} \delta\left(\mathbf{x}-\mathbf{x}_{m}\right) \\
\Delta p^{(3)}(\mathbf{x})+k_{0}^{2} p^{(3)}(\mathbf{x})=-F_{n_{d}}^{(3)} \frac{\partial}{\partial \boldsymbol{n}_{d}} \delta\left(\mathbf{x}-\mathbf{x}_{d}\right)[24]
\end{gathered}
$$

where $\omega$ is the angular frequency, $Q_{v}$ the volume velocity flow of the monopole source, $F_{n_{d}}^{(3)}=j \omega \rho_{0} d Q_{v}^{(3)}$ the dipole source strength (or dipole force) and $k_{0}=\omega / c_{0}$ the acoustic wavenumber. The equilibrium equation for the structure in each case is

$$
\begin{gathered}
L v^{(1)}(\tilde{\mathbf{x}})=F^{(1)} \delta\left(\tilde{\mathbf{x}}-\tilde{\mathbf{x}}_{f}\right)-p^{(1)}(\tilde{\mathbf{x}}), \\
L v^{(2)}(\tilde{\mathbf{x}})=-p^{(2)}(\tilde{\mathbf{x}}) \\
L v^{(3)}(\tilde{\mathbf{x}})=-p^{(3)}(\tilde{\mathbf{x}})
\end{gathered}
$$

where $L$ is the self-adjoint operator of the structure and $v$ is the structure velocity in direction $\boldsymbol{n}$ normal to the structure. Euler's formula provides a relation between the pressure gradient in the fluid and the velocity along the normal external to the fluid. On the surface of the structure, the normal external to the fluid corresponds to $-\boldsymbol{n}$. In this particular case, Euler's formula becomes

$$
\frac{\partial p}{\partial \boldsymbol{n}}(\tilde{\mathbf{x}})=j \omega \rho_{0} v(\tilde{\mathbf{x}})
$$

\section{Reciprocity principle for the radiated pressure (monopole source)}

Multiplying Eq. (A1) by $p^{(2)}(\mathbf{x})$ and Eq. (A2) by $-p^{(1)}(\mathbf{x})$, adding them and integrating them over the entire acoustic domain, one obtains

$$
\int_{V}\left[\Delta p^{(1)}(\mathbf{x}) p^{(2)}(\mathbf{x})-\Delta p^{(2)}(\mathbf{x}) p^{(1)}(\mathbf{x})\right] d \mathbf{x}=j \omega \rho_{0} Q_{v}^{(2)} \int_{V} p^{(1)}(\mathbf{x}) \delta\left(\mathbf{x}-\mathbf{x}_{m}\right) d \mathbf{x} .
$$

The volume integral on the left-hand side of Eq. (A8) can be transformed to a surface integral using Green's theorem. Then, using Euler's formula together with Eqs. (A4) and (A5), 
one finally obtains [18]

$$
F^{(1)} \int_{S} \frac{\partial p^{(2)}}{\partial \boldsymbol{n}}(\tilde{\mathbf{x}}) \delta\left(\tilde{\mathbf{x}}-\tilde{\mathbf{x}}_{f}\right) d \tilde{\mathbf{x}}=j \omega \rho_{0} Q_{v}^{(2)} \int_{V} p^{(1)}(\mathbf{x}) \delta\left(\mathbf{x}-\mathbf{x}_{m}\right) d \mathbf{x}
$$

From the property of the Dirac delta function (i.e., $\int_{V} f(\mathbf{x}) \delta\left(\mathbf{x}-\mathbf{x}_{0}\right) d \mathbf{x}=f\left(\mathbf{x}_{0}\right)$ for any function $f$ defined on $V$ and any point $\mathbf{x}_{0} \in V$ ) one has:

$$
F^{(1)} \frac{\partial p^{(2)}}{\partial \boldsymbol{n}}\left(\tilde{\mathbf{x}}_{f}\right)=j \omega \rho_{0} Q_{v}^{(2)} p^{(1)}\left(\mathbf{x}_{m}\right)
$$

Using Eq. (A7), Eq. (A10) becomes

$$
\frac{p^{(1)}}{F^{(1)}}\left(\mathbf{x}_{m}\right)=\frac{v^{(2)}}{Q_{v}^{(2)}}\left(\tilde{\mathbf{x}}_{f}\right)
$$

Eq. (A11) shows that the pressure radiated by a structure at point $\mathbf{x}_{m}$ when it is excited by a normal unit point force at point $\tilde{\mathbf{x}}_{f}$ equals the structure normal velocity at point $\tilde{\mathbf{x}}_{f}$ when it is excited by a monopole source of unit volume velocity at point $\mathbf{x}_{m}$.

\section{Reciprocity principle for the particle velocity (dipole source)}

Multiplying Eq. (A1) by $p^{(3)}(\mathbf{x})$ and Eq. (A3) by $-p^{(1)}(\mathbf{x})$, adding them and integrating them over the entire acoustic domain, one obtains

$$
\int_{V}\left[\Delta p^{(1)}(\mathbf{x}) p^{(3)}(\mathbf{x})-\Delta p^{(3)}(\mathbf{x}) p^{(1)}(\mathbf{x})\right] d \mathbf{x}=F_{n_{d}}^{(3)} \int_{V} p^{(1)}(\mathbf{x}) \frac{\partial}{\partial \boldsymbol{n}_{d}} \delta\left(\mathbf{x}-\mathbf{x}_{d}\right) d \mathbf{x} .
$$

As previously, the volume integral on the left-hand side of Eq. (A12) can be transformed to a surface integral using Green's theorem. Then, using Euler's formula together with Eqs. (A4) and (A6), one finally obtains [18]

$$
F^{(1)} \int_{S} \frac{\partial p^{(3)}}{\partial \boldsymbol{n}}(\tilde{\mathbf{x}}) \delta\left(\tilde{\mathbf{x}}-\tilde{\mathbf{x}}_{f}\right) d \tilde{\mathbf{x}}=F_{n_{d}}^{(3)} \int_{V} p^{(1)}(\mathbf{x}) \frac{\partial}{\partial \boldsymbol{n}_{d}} \delta\left(\mathbf{x}-\mathbf{x}_{d}\right) d \mathbf{x}
$$

Using the previously described property of the Dirac delta function and the property of the distributional derivative of the Dirac delta function (i.e., $\int_{V} f(\mathbf{x}) \frac{\partial}{\partial \boldsymbol{n}} \delta\left(\mathbf{x}-\mathbf{x}_{0}\right) d \mathbf{x}=$ $-\int_{V} \frac{\partial}{\partial \boldsymbol{n}} f(\mathbf{x}) \delta\left(\mathbf{x}-\mathbf{x}_{0}\right) d \mathbf{x}=-\frac{\partial}{\partial \boldsymbol{n}} f\left(\mathbf{x}_{0}\right)$ for any function $f$ defined on $V$ and any point 
685

686

$\left.\mathbf{x}_{0} \in V\right)$ Eq. (A13) becomes

$$
F^{(1)} \frac{\partial p^{(3)}}{\partial \boldsymbol{n}}\left(\tilde{\mathbf{x}}_{f}\right)=-F_{n_{d}}^{(3)} \frac{\partial p^{(1)}}{\partial \boldsymbol{n}_{d}}\left(\mathbf{x}_{d}\right)
$$

Using Euler's formula, the normal velocity of the structure at point $\tilde{\mathbf{x}}_{f}$ and the particle velocity $v_{n_{d}}$ in direction $\boldsymbol{n}_{d}$ at point $\mathbf{x}_{d}$ are introduced in Eq. (A14). One finally obtains

$$
\frac{v_{n_{d}}^{(1)}}{F^{(1)}}\left(\mathbf{x}_{d}\right)=\frac{v^{(3)}}{F_{n_{d}}^{(3)}}\left(\tilde{\mathbf{x}}_{f}\right) .
$$

Eq. (A15) shows that the particle velocity in direction $\boldsymbol{n}_{d}$ at point $\mathbf{x}_{d}$ in the acoustic medium when the structure is excited by a normal unit point force at point $\tilde{\mathbf{x}}_{f}$ equals the structure normal velocity at point $\tilde{\mathbf{x}}_{f}$ when it is excited by a dipole source at point $\mathbf{x}_{d}$ oriented in direction $\boldsymbol{n}_{d}$ and of unit injected force.

\section{Appendix B: Numerical simulations}

The panel vibratory response should be estimated numerically for the four different cases of excitation: (a) a wall plane wave for $H_{\alpha}(\mathbf{x}, \boldsymbol{k}, \omega)$; (b) a normal point force for $H_{v / F_{n}}(\tilde{\mathbf{x}}, \mathbf{x}, \omega)$; (c) a monopole source for $H_{v / Q_{v}}(\tilde{\mathbf{x}}, \mathbf{x}, \omega)$; (d) a dipole source for $H_{v / F_{0}}(\tilde{\mathbf{x}}, \mathbf{x}, \omega)$. They can be estimated by neglecting the fluid-structure interaction and considering the modal expansion technique. For a panel that has simply supported boundary conditions on all of its edges, the modal angular frequency $\omega_{m n}$, the spatial mode shape $\phi_{m n}$, and the modal mass $M_{m n}$ for the $(m, n)$ mode are given, respectively, by

$$
\omega_{m n}=\left[\left(\frac{m \pi}{L_{x}}\right)^{2}+\left(\frac{n \pi}{L_{y}}\right)^{2}\right] \sqrt{\frac{D}{\rho h}},
$$

$$
\phi_{m n}(\mathbf{x})=\sin \left(\frac{m \pi}{L_{x}} x\right) \sin \left(\frac{n \pi}{L_{y}} y\right),
$$

$$
M_{m n}=\frac{\rho h L_{x} L_{y}}{4},
$$

where $m$ and $n$ are non-zero strictly positive integers. The normal velocity $v$ of the plate 
excited by the pressure distribution $P(\tilde{\mathbf{x}}), \tilde{\mathbf{x}} \in \Sigma_{p}$ is then obtained with:

$$
v(\mathbf{x}, \omega)=j \omega \sum_{m, n} \frac{\boldsymbol{F}_{m n} \phi_{m n}(\mathbf{x})}{M_{m n}\left(\omega_{m n}^{2}-\omega^{2}+j \eta \omega \omega_{m n}\right)},
$$

where the modal force $\boldsymbol{F}_{m n}$ is defined by

$$
\boldsymbol{F}_{m n}=\int_{\Sigma_{p}} P(\tilde{\mathbf{x}}) \phi_{m n}(\tilde{\mathbf{x}}) d \tilde{\mathbf{x}}
$$

\section{Calculation of the sensitivity functions with the direct interpretation}

The direct interpretation described in Sec. III and in Fig. 2 indicates that the sensitivity functions are equal to the system response at point $\mathbf{x}$ when the panel is excited by wall-pressure plane waves of wavenumber $-\boldsymbol{k}=\left(-k_{x},-k_{y}\right)$. The modal force is therefore calculated by considering the pressure distribution $P(\tilde{\mathbf{x}})=e^{-j \boldsymbol{k} \tilde{\mathbf{x}}}$. In this case, the analytical solution to Eq. (B5) is

$$
\boldsymbol{F}_{m n}=I_{m}^{x} I_{n}^{y}
$$

where for $\xi=x$ or $\xi=y$,

$$
I_{p}^{\xi}=\left\{\begin{array}{l}
\left(\frac{p \pi}{L_{\xi}}\right) \frac{(-1)^{p} e^{-j k_{\xi} L_{\xi}}-1}{k_{\xi}^{2}-\left(\frac{p \pi}{L_{\xi}}\right)^{2}}, \text { if }\left|k_{\xi}\right| \neq \frac{p \pi}{L_{\xi}} \\
\frac{1}{2} j L_{\xi}, \text { otherwise. }
\end{array}\right.
$$

The sensitivity functions $H_{v}$ for a point $\mathbf{x}$ on the panel can be directly estimated using Eqs. (B4) to (B7). Based on the velocity response of the plate to a wall-pressure plane wave, the radiated pressure at a point $\mathbf{x}$ in the acoustic domain may be calculated using the Rayleigh integral [23] whereas the particle velocity may be deduced from the Euler equation. Doing so for a set of wall-pressure plane waves allows calculating the sensitivity functions $H_{p}$ and $H_{v 0}$ at point $\mathbf{x}$ in the acoustic domain.

\section{Calculation of the sensitivity functions with the reciprocity interpretation}

The calculation of the velocity sensitivity function $H_{v}$ at point $\mathbf{x}$ using the reciprocity principle involves exciting the plate with a normal unit force at point $\mathbf{x}$. The modal force is 
thus simply given by

$$
\boldsymbol{F}_{m n}=\phi_{m n}(\mathbf{x}) .
$$

According to the process described in Sec. V, the normal velocity of the panel should be calculated with Eq. (B4) for points $\tilde{\mathbf{x}} \in \Gamma_{\tilde{\mathbf{x}}}$. A discrete Fourier transform is then applied to deduce the sensitivity functions $H_{v}(\mathbf{x}, \boldsymbol{k}, \omega)$.

Similarly, the pressure sensitivity function $H_{p}$ at point $\mathbf{x}$ in the acoustic domain is obtained by exciting the plate by a monopole at point $\mathbf{x}$. The particle velocity sensitivity function $H_{v_{0}}$ at point $\mathbf{x}$ is obtained analogously using a dipole excitation in the direction $\boldsymbol{n}$ at point $\mathbf{x}$. The modal force is then obtained by approximating the integral of Eq. (B5) with the rectangular integration rule for a wall-pressure defined by

$$
P(\tilde{\mathbf{x}})=j \omega \rho_{0} Q_{v} \frac{e^{-j k_{0} r}}{2 \pi r}
$$

for the monopole case, with $r=|\mathbf{x}-\tilde{\mathbf{x}}|$ and $Q_{v}=1 \mathrm{~m}^{3} \cdot \mathrm{s}^{-1}$. For the dipole case,

$$
P(\tilde{\mathbf{x}})=\frac{F_{0}}{d}\left(\frac{e^{-j k_{0} r_{1}}}{2 \pi r_{1}}-\frac{e^{-j k_{0} r_{2}}}{2 \pi r_{2}}\right) .
$$

where $r_{1}$ and $r_{2}$ are two positions of monopoles separated by a distance $d$ representing a dipole at a distance $r$ [24] and the dipole force will be then considered of unit amplitude (i.e., $\left.F_{0}=1 \mathrm{~N}\right)$. 
Fig. 1. Panel (gray line) and coordinate system. (a) receiving side: semi-infinite domain. (b) source side: theoretical DAF.

Fig. 2. Direct interpretation of the sensitivity functions: (a) $H_{v}$, (c) $H_{p}$, (e) $H_{v_{0}}$ and corresponding reciprocal interpretation (b) $H_{v}$, (d) $H_{p}$, (f) $H_{v_{0}}$. (d) and (f) see appendix for demonstration.

Fig. 3. Experimental setup. (a) plate excited by a shaker to determine $H_{v}$. (b) and (c) baffled plate excited by a monopole source to determine $H_{p}$ and $H_{v_{0}}$. 1 - shaker with impedance head. 2 - plate. 3 - frame. 4 - baffle. 5 - sound absorbing foam. 6 monopole source mounted on 3-axis robot. 7 - single-point laser vibrometer mounted on 2-axis robot.

Fig. 4. Velocity sensitivity functions at point $\mathbf{x}_{M},\left|H_{v}\right|^{2}\left(\mathrm{~dB}\right.$, ref. $\left.1 \mathrm{~m}^{2} \cdot \mathrm{s}^{-2}\right)$ : direct calculation (left), numerical reciprocity approach (middle), experimental reciprocity approach (right). (a) $f=178 \mathrm{~Hz}$. (b) $f=600 \mathrm{~Hz}$. (c) $f=1710 \mathrm{~Hz}$. - - , circle of radius $k_{f} .-$, circle of radius $k_{0}$.

Fig. 5. Product of sensitivity functions at point $\mathbf{x}_{N},\left|\operatorname{Re}\left\{H_{p} \times H_{v_{0}}^{*}\right\}\right|(\mathrm{dB}$, ref. 1 Pa.m.s ${ }^{-1}$ ): direct calculation (left), numerical reciprocity approach (middle), experimental reciprocity approach (right). (a) $f=178 \mathrm{~Hz}$. (b) $f=600 \mathrm{~Hz}$. (c) $f=1710 \mathrm{~Hz}$. - - -, circle of radius $k_{f}$. — , circle of radius $k_{0}$.

Fig. 6. Velocity ASD functions $G_{v v}\left(\mathrm{~dB}\right.$, ref. $\left.1 \mathrm{~m}^{2} \cdot \mathrm{s}^{-2} \cdot \mathrm{Hz}^{-1}\right)$. (a) direct calculation (bold gray line) vs. numerical reciprocity approach (light black line). (b) numerical reciprocity approach (bold gray line) vs. experimental reciprocity approach (light black line).

Fig. 7. Pressure - particle velocity CSD functions $\operatorname{Re}\left\{G_{p v_{0}}\right\}\left(\mathrm{dB}\right.$, ref. $\left.1 \mathrm{~W}^{2} \cdot \mathrm{m}^{-2} \cdot \mathrm{Hz}^{-1}\right)$. (a) direct calculation (bold gray line) vs. numerical reciprocity approach (light black line). (b) numerical reciprocity approach (bold gray line) vs. experimental reciprocity approach (light black line).

Fig. 8. Velocity ASD functions $G_{v v}\left(\mathrm{~dB}\right.$, ref. $\left.1 \mathrm{~m}^{2} \cdot \mathrm{s}^{-2} \cdot \mathrm{Hz}^{-1}\right)$ : reverberant room measurements (bold gray line) vs. experimental reciprocity approach (light black line). 
Fig. 9. Illustration of the virtual surface $\Sigma_{v}$ considered for estimating the radiated power. (a) in the transmission loss facility. (b) with the reciprocal approach. (c) with the reciprocal approach considering the symmetry properties of the system.

Fig. 10. Transmission loss (dB). (a) numerical approach (bold gray line) vs. experimental reciprocity approach (light black line). (b) transmission loss facility (bold gray line) vs. experimental reciprocity approach (light black line).

Fig. 11. Illustration of the three considered vibroacoustic problems of an elastic structure excited by: (a) a normal force at point $\tilde{\mathbf{x}}_{f}$, (b) a monopole source at point $\mathbf{x}_{m}$ (internal or external to the structure), (c) a dipole source at point $\mathbf{x}_{d}$ (internal or external to the structure). 
TABLE I. Properties of the simply supported aluminum plate.

\begin{tabular}{cc}
\hline \hline Parameter (Symbol), Unit & Value \\
\hline Young's modulus $(E), \mathrm{GPa}$ & 68.9 \\
Poisson's ratio $(\nu)$ & 0.3 \\
Mass density $(\rho), \mathrm{kg} / \mathrm{m}^{3}$ & 2740 \\
Length $\left(L_{x}\right), \mathrm{mm}$ & 480 \\
Width $\left(L_{y}\right), \mathrm{mm}$ & 420 \\
Thickness $(h), \mathrm{mm}$ & 3.17 \\
\hline \hline
\end{tabular}

